\title{
Antiferromagnetic coupling in fcc Fe overlayers on $\mathrm{Ni} / \mathrm{Cu}(100)$
}

\author{
B. Schirmer \\ Institut für Grenzflächenforschung und Vakuumphysik, Forschungszentrum Jülich, D-52428 Jülich, Germany \\ M. Wuttig \\ I. Physikalisches Institut der RWTH Aachen, D-52056 Aachen, Germany \\ and Institut für Grenzflächenforschung and Vakuumphysik, Forschungszentrum Jülich, D-52428 Jülich, Germany
}

(Received 14 April 1999)

\begin{abstract}
Magnetic properties of ultrathin fcc Fe overlayers on $\mathrm{Ni} / \mathrm{Cu}(100)$ have been determined to study the influence of a magnetic interface. Three regions of different magnetic behavior are distinguished by magneto-optic Kerr ellipsometry, in line with previous studies of $\mathrm{Fe} / \mathrm{Co} / \mathrm{Cu}(100)$ and $\mathrm{Fe} / \mathrm{Ni} / \mathrm{Cu}(100)$. These magnetic states are closely related to the film structure. Above 10 monolayers (ML), the iron films are homogeneously magnetized and adopt the bcc phase. Very thin films up to $2.5 \mathrm{ML}$ are homogeneously magnetized as well but show an fcc structure in conjunction with a $(4 \times 1)$ reconstruction. The most complex magnetic properties characterize Fe films between 5 and $10 \mathrm{ML}$. In this thickness range the iron films are not homogeneously magnetized. Instead ferromagnetism is only observed at the Fe film surface and the Fe/Ni film interface. The surface magnetization is apparently correlated with an enlarged atomic volume at the surface and a $(2 \times 1)$ surface reconstruction. Additionally, the magnetic Ni substrate induces ferromagnetic order in the Fe film at the $\mathrm{Fe} / \mathrm{Ni}$ interface. The coupling of the two ferromagnetic portions of the film shows a strong temperature dependence. This is attributed to the temperature dependence of the bilinear and biquadratic exchange coupling. At low temperature an antiferromagnetic coupling between the two ferromagnetic portions is observed. With increasing temperature this is followed by a canted spin arrangement and finally a ferromagnetic coupling. [S0163-1829(99)07041-1]
\end{abstract}

\section{INTRODUCTION}

The discovery of giant magnetoresistence ${ }^{1}$ and the potential impact of magnetic random access memories have spurred a surge of research into magnetism of thin films and interfaces. Tailoring of magnetic properties of multilayers is one of the prime research goals. Different approaches have been adopted to achieve this aim including a tailoring of film structures and the related magnetic properties.

Ultrathin iron films grown on $\mathrm{Cu}(100)$ exhibit a rich variety of structural and magnetic phases. ${ }^{2-12}$ With increasing iron film thickness three different structural modifications are observed. Up to $11 \mathrm{ML} \mathrm{Fe}$, two different fcc phases are stabilized. The first one exists up to $5 \mathrm{ML}$ and is characterized by ferromagnetic order, an enlarged atomic volume of $12.1 \AA^{3}$, and a particular reconstruction pattern indicative of structural instability. ${ }^{3}$ For Fe films between 5 and $11 \mathrm{ML}$, only the first two layers show an enlarged atomic volume of $12.1 \AA^{3}$ and ferromagnetism. ${ }^{5-7}$ The interior of the film has an atomic volume of $11.4 \AA^{3}$ and presumably shows antiferromagnetic interlayer coupling ${ }^{8}$. The transition to the stable bcc phase of iron is observed above $11 \mathrm{ML}^{9-11}$ The transitions between the structural and magnetic phases depend both upon growth temperature and base pressure.,13

Such a situation is ideal to explore the possible role of a magnetic interface on the structure and magnetism of thin films. Indeed, a number of interesting observations have been made for $\mathrm{Fe}$ films deposited on either Co or Ni films on $\mathrm{Cu}(100) .{ }^{14-18}$ O'Brien and Tonner studied Fe films on $\mathrm{Ni}$ and $\mathrm{Co}$ on $\mathrm{Cu}(100)$ (Refs. 14,15) and found a magnetic behavior at room temperature which closely resembles the be- havior of $\mathrm{Fe} / \mathrm{Cu}(100)$. Below $5 \mathrm{ML}$, the iron films couple ferromagnetically on $\mathrm{Ni} / \mathrm{Cu}(100)$. Between 5 and $11 \mathrm{ML}$, the $\mathrm{Fe}$ films only possess a ferromagnetic live layer at the $\mathrm{Fe} / \mathrm{Ni}$ interface but none at the surface. ${ }^{14}$ In this thickness range, $\mathrm{Fe}$ films on $\mathrm{Cu}(100)$ showed magnetically live layers at the surface below $270 \mathrm{~K}$ which were correlated to an enlarged atomic volume and a $(2 \times 1)$ reconstruction at the surface. We have studied the growth and structure of $\mathrm{Fe}$ on $\mathrm{Ni} / \mathrm{Cu}(100) .{ }^{19}$ In particular between 5 and $11 \mathrm{ML}$, the films showed a $(2 \times 1)$ surface reconstruction and a quantitative low-energy electron diffraction (LEED) $\mathrm{I}(\mathrm{V})$ analysis revealed an enlarged atomic volume of $12.1 \AA^{3}$ at the film surface while the film interior had an atomic volume of $11.4 \AA^{3} .{ }^{19}$ Based on the close correlation between structure and magnetism, the $\mathrm{Fe}$ films on $\mathrm{Ni} / \mathrm{Cu}(100)$ should also have magnetically live surface layers. Measurements at room temperature did not find any evidence for surface magnetism for $\mathrm{Fe} / \mathrm{Co} / \mathrm{Cu}(100)$ and $\mathrm{Fe} / \mathrm{Ni} / \mathrm{Cu}(100) .{ }^{14-17}$ Instead in both cases magnetic Fe layers at the interface were observed. Recently, Schmitz et al. ${ }^{18}$ investigated magnetism of $\mathrm{Fe} / \mathrm{Co} / \mathrm{Cu}(100)$ at $110 \mathrm{~K}$ and room temperature. These measurements confirm the existence of a magnetic layer at the interface. In addition, the magnetic circular dichroism data reveal that the Fe film surface is ferromagnetic as well at $110 \mathrm{~K}$. Both ferromagnetic $\mathrm{Fe}$ films couple ferromagnetically to each other at low temperatures.

In this paper we present our data for the magnetic properties of $\mathrm{Fe}$ films on $\mathrm{Ni} / \mathrm{Cu}(100)$. Particular emphasis is placed on the film thickness range between 5 and $11 \mathrm{ML} \mathrm{Fe}$, where we expect to find magnetically live surface layers. Temperature dependent measurements were performed to 
obtain deeper insight into the magnetic order and coupling of the Fe films. In the next section we give a short description of the experimental setup. In the third section the results of the magnetic investigations of the $\mathrm{Fe} / \mathrm{Ni} / \mathrm{Cu}(100)$ system are presented. In Sec. IV a discussion and comparison with previous results can be found. The last section contains a short summary.

\section{EXPERIMENT}

The experiments were performed in an ultrahigh vacuum chamber equipped with several facilities for preparation and characterization of thin films including Auger electron spectroscopy (AES), medium energy electron diffraction (MEED), LEED, and magneto-optic Kerr ellipsometry (MOKE). Only a brief description of the system, the preparation of the sample, and the characterization of the growth and the film structure will be given here because the apparatus and the sample treatment have already been presented elsewhere. ${ }^{20}$ The results of our growth and structure investigation are found in Ref. 19. The base pressure of the chamber is $6 \times 10^{-9} \mathrm{~Pa}$. Prior to film deposition the copper (100) crystal was cleaned by $\mathrm{Ar}^{+}$sputter and annealing cycles. The films of iron and nickel were deposited from small disks of high purity (Fe $99.99 \%$, Ni 99.98\%) by thermal evaporation with a typical evaporation rate of $0.3 \mathrm{ML} / \mathrm{min}$. During the evaporation the residual gas pressure was below 2 $\times 10^{-8} \mathrm{~Pa}$. The thickness of the films was controlled by AES and MEED oscillations which allow a high precision in thickness determination. Most of the samples were deposited with a wedgelike thickness variation. The thickness profile of such films was determined by the Auger electron intensity ratio. Using wedges not only warrants identical growth conditions for different film thicknesses but also allows the precise determination of thicknesses where magnetic properties are changing.

During the deposition of the Ni film the sample was kept at $350 \mathrm{~K}$ to improve the film quality. Fe was subsequently deposited at a sample temperature of $300 \mathrm{~K}$. For the MOKE measurements a He-Ne Laser with a wavelength of $632.8 \mathrm{~nm}$ was used as a light source. While a few test measurements were also recorded in polar geometry most hysteresis loops were recorded in the longitudinal direction with an angle of incidence of $65^{\circ}$ with respect to the surface normal. These two different geometries differ by a factor of 6.3 in sensitivity. ${ }^{21,22}$ The Kerr effect was measured employing a null ellipsometer with polarizer-sample-compensatoranalyzer arrangement. A maximum field of 500 Oe could be applied which was aligned parallel to the fcc[001] direction. This is the easy axis of the system. Most hysteresis loops were recorded at a sample temperature of $110 \mathrm{~K}$ but measurements were also performed up to $400 \mathrm{~K}$.

\section{RESULTS}

To be able to subtract the contribution of the Ni films from the magnetic signal, MOKE data were measured for 7 ML Ni on $\mathrm{Cu}(100)$. In polar geometry no magnetic response was measured for applied fields up to $500 \mathrm{Oe}$. In longitudinal geometry a small hysteresis is observed [Fig. 1(a)]. A small saturation magnetization $\left(M_{s}\right)$ of $3.1 \pm 1.4 \mu \mathrm{rad}$ and a weak
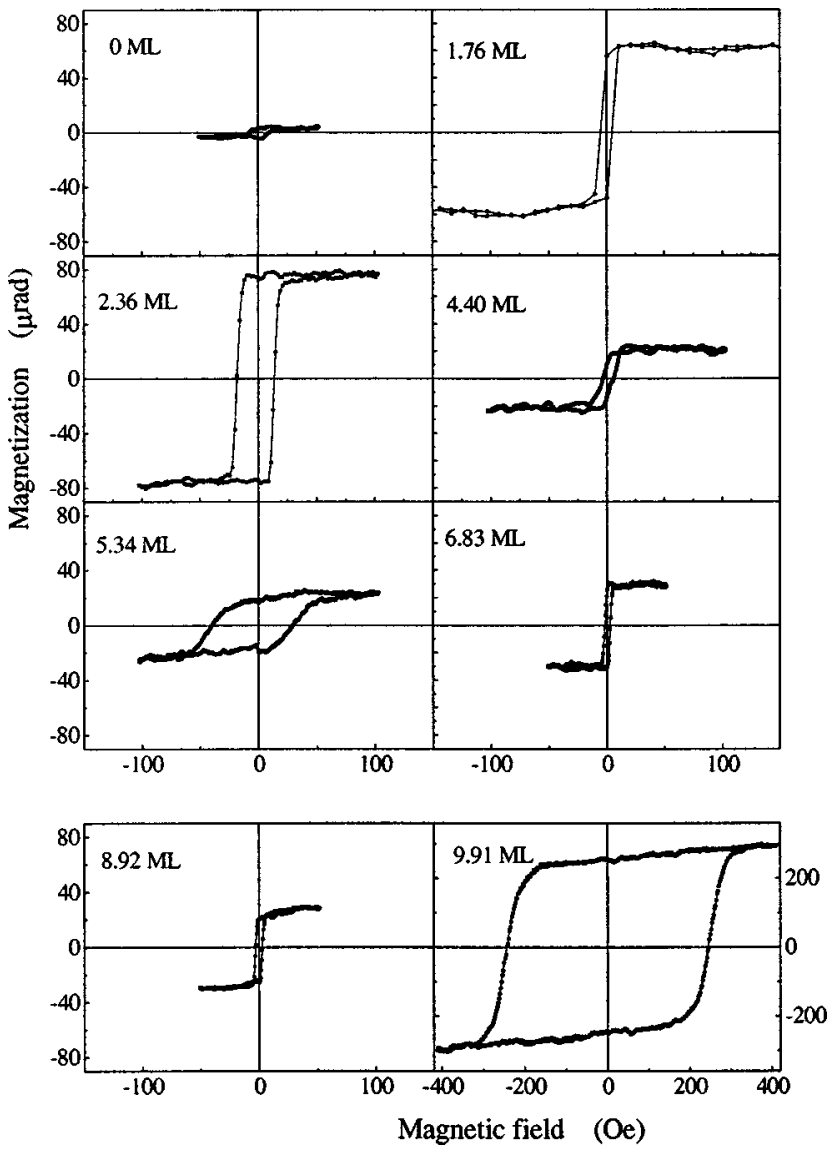

FIG. 1. Longitudinal MOKE hysteresis loops of the system Fe/ $\mathrm{Ni} / \mathrm{Cu}(100)$ in the Fe thickness range between 0 and 9.91 ML Fe measured at $110 \mathrm{~K}$. The Ni thickness is always $7 \mathrm{ML}$. The last loop measured for 9.91 ML Fe has been rescaled.

coercive field of 8.8 Oe are found. Due to the low value for $M_{s}$, the data show considerable scatter. The weak signal is not only caused by the smaller magnetic moment of $\mathrm{Ni}$ compared with $\mathrm{Co}$ and $\mathrm{Fe}$. It is also caused by the rather weak magneto-optic interaction of $\mathrm{Ni}$. The results described above are in line with previous findings. A coercive field of approximately 20 Oe has been reported by $\mathrm{Wu}$ et al. ${ }^{23}$ and of approximately 7.5 Oe by O'Brien, Drobay, and Tonner. ${ }^{14}$ The spin-reorientation transition for $\mathrm{Ni} / \mathrm{Cu}(100)$ has been observed by $\mathrm{Wu}$ et al. $^{23}$ and Bochi et al. ${ }^{24}$ to occur between 7 and $8 \mathrm{ML}$ of $\mathrm{Ni}$. The saturation magnetization below the transition is approximately $5 \mu \mathrm{rad},{ }^{23}$ in reasonable agreement with our finding.

A large number of hysteresis loops was recorded at $110 \mathrm{~K}$ for Fe layer thicknesses up to $10 \mathrm{ML}$ deposited on $7 \mathrm{ML} \mathrm{Ni}$ on $\mathrm{Cu}(100)$. A selection of these data is shown in Fig. 1. The first two hysteresis loops were taken at 1.76 and $2.36 \mathrm{ML}$. Both curves have a rectangular shape. The magnetization signal increases from nearly 60 to $80 \mu \mathrm{rad}$. The next four loops show the thickness range between 4.4 and 8.92 ML. They have a reduced $M_{s}$ signal in comparison with the first two loops. The $M_{s}$ values are nearly constant and lie between 25 and $30 \mu \mathrm{rad}$. The coercive field is also nearly constant at a low level, with the exception of the 5.34 ML thick Fe film, which exhibits a larger value (Fig. 1). The last loop of Fig. 1 shows a typical hysteresis curve for the Fe thickness range above 9.7 ML. In this thickness region both the 


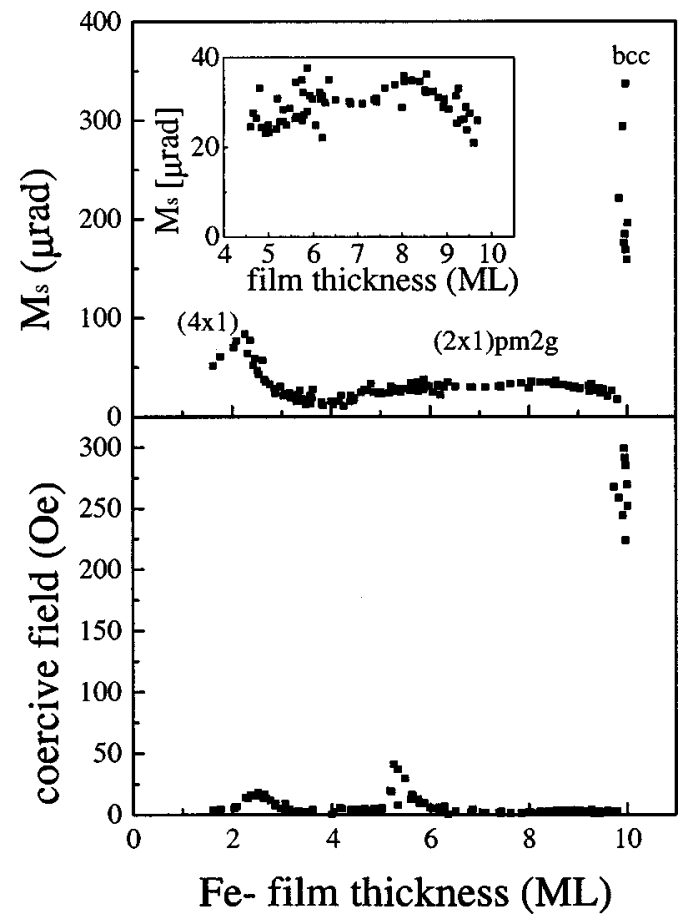

FIG. 2. Thickness dependence of the Kerr ellipticity at saturation $\left(M_{s}\right)$ and the coercive field $\left(H_{c}\right)$ for the system $\mathrm{Fe} / \mathrm{Ni} / \mathrm{Cu}(100)$. The inset in the upper diagram shows a magnification of the saturation magnetization for the Fe thickness range between 4.5 and 10 ML. The data have been measured at $110 \mathrm{~K}$.

saturation magnetization and the coercive field increase dramatically. Please note that both axes in the plot (Fig. 1) have been rescaled in comparison with the other diagrams. The form of the loop is nearly rectangular with only a small difference between the saturation magnetization and the remanence.

The saturation magnetization $M_{s}$ and the coercive field $H_{c}$ are plotted against the film thickness in Fig. 2. This presentation shows three different regimes of magnetic behavior. The corresponding film structure is depicted as well. In the thickness regime up to approximately $2.5 \mathrm{ML}$, the saturation magnetization increases linearly. This is indicative for a homogeneously magnetized Fe film. For these thicknesses a $(4 \times 1)$ reconstruction is observed. After a decrease of magnetization with increasing thickness a rather constant magnetization is observed between 4.5 ML and 9.7 ML, which is accompanied by an fcc phase with $(2 \times 1)$ surface reconstruction. Above 9.7 ML the magnetization increases drastically and quickly reaches values of $300 \mu \mathrm{rad}$ around 10 ML. Similar magnetization values have also been recorded for $\mathrm{Fe} / \mathrm{Cu}(100)$. The coercive field also increases drastically upon the phase transition to bcc iron and shows a maximum coercivity around 300 Oe. Much weaker maxima are observed for smaller thicknesses. A first maximum occurs around 2.5 ML, where we have previously observed the phase transformation from a $(4 \times 1)$ phase at small thicknesses to a $(2 \times 1)$ structure at larger thickness. A second maximum is observed around 5.3 ML. The inset in Fig. 2 shows the enlarged behavior of $M_{s}$ between 4.5 and $10 \mathrm{ML}$ Fe. This is the thickness regime we are most interested in, since for these films we expect both ferromagnetically live surface and interface layers. This is in line with the constant

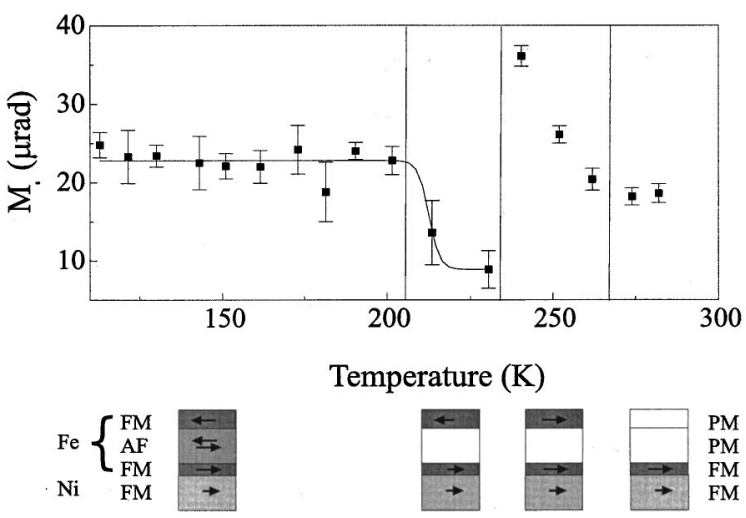

FIG. 3. Temperature dependence of the Kerr ellipticity at saturation of the $\mathrm{Fe} / \mathrm{Ni} / \mathrm{Cu}(100)$ system for a $5.3 \mathrm{ML}$ thick Fe film. In the bottom a model for the temperature dependence of the magnetization of the 5.3 ML thick film. Four regions with different magnetic coupling are displayed. The second region is characterized by a canted spin arrangement where the magnetic moments of the ferromagnetic Fe surface layer and the ferromagnetic Fe/Ni interface are no longer collinear. This is indicated by the reduced length of the arrows in this thickness range, which describes the component of magnetization parallel to the applied field.

magnetization observed in this thickness regime. On the other hand, we only observe a rather low value for $M_{s}$. Comparing the magnetization of $30 \pm 5 \mu \mathrm{rad}$ in this range with the homogeneously magnetized films at $2.4 \mathrm{ML}$ $(\approx 80 \mu \mathrm{rad})$ and at $10 \mathrm{ML}(\approx 300 \mu \mathrm{rad})$ implies that less than $1 \mathrm{ML}$ is ferromagnetic, assuming a similar magnetic moment compared with films in regions I and III.

To obtain a better understanding of this behavior we have measured the temperature dependence of the saturation for a 5.3 ML thick film. The corresponding data up to $300 \mathrm{~K}$ are displayed in Fig. 3. Interestingly enough, in this figure four different regions are clearly visible. In the first range up to approximately $210 \mathrm{~K}$, the Kerr ellipticity remains nearly constant at a level of $\approx 23 \mu \mathrm{rad}$. In the second temperature range between 210 and $230 \mathrm{~K}$, a clear decrease in $M_{s}$ is observed down to $9 \mu \mathrm{rad}$. Between 230 and $240 \mathrm{~K}$, a sharp jump in the signal is visible. At the temperature of $240 \mathrm{~K}$ the ellipticity signal has increased to $36 \mu \mathrm{rad}$. In the third range between 240 and $270 \mathrm{~K}$, the magnetization shows a strong decrease down to a saturation level of $18 \mu \mathrm{rad}$. It remains nearly constant for the last two data points measured above $270 \mathrm{~K}$. This temperature dependence gives evidence for a complex magnetic order of the iron films.

\section{DISCUSSION}

Comparing the magnetic behavior of $\mathrm{Fe} / \mathrm{Ni} / \mathrm{Cu}(100)$ with previous measurements for $\mathrm{Fe} / \mathrm{Cu}(100)$ shows pronounced similarities but also interesting differences. In both cases three regimes with different structural and magnetic properties are observed. Above $10 \mathrm{ML} \mathrm{Fe}$, a bcc phase is observed which is homogeneously magnetized and shows in-plane magnetization on $\mathrm{Cu}(100)$ and $\mathrm{Ni} / \mathrm{Cu}(100)$. Below this thickness, a broad regime exists where the magnetization is small and constant on both substrates. While the magnetization is perpendicular to the surface on $\mathrm{Cu}(100)$, an in-plane magnetization is found on $\mathrm{Ni} / \mathrm{Cu}(100)$. This is related to the mag- 
netization of the underlying Ni film. O'Brien, Doubray, and Tonner have deposited $\mathrm{Fe}$ on slightly thicker Ni films which exhibit perpendicular magnetization caused by a straininduced spin reorientation. ${ }^{14}$ In their case, a perpendicular magnetization of the iron film is observed. This implies that the magnetization direction of the iron film is controlled by the magnetic anisotropy of the underlying Ni film.

For low Fe film thicknesses a third phase exists. It is characterized by a homogeneous magnetization in the entire film and is accompanied by a $(4 \times 1)$ superstructure on $\mathrm{Ni} /$ $\mathrm{Cu}(100)$. On $\mathrm{Cu}(100)$, this iron phase shows a $(4 \times 1)$ and $(5 \times 1)$ reconstruction. While this phase only exists up to 2.5 ML on $\mathrm{Ni} / \mathrm{Cu}(100)$, it can be observed up to $4 \mathrm{ML}$ on $\mathrm{Cu}(100)$. The magnetization of this phase is perpendicular on $\mathrm{Cu}(100)$ and in plane on $\mathrm{Ni} / \mathrm{Cu}(100)$ for the $\mathrm{Ni}$ film thickness we have chosen. Again this is attributed to the influence of the magnetization direction of the Ni film. The differences in magnetic anisotropy of the films make a quantitative comparison of magnetization levels difficult. The measured Kerr signal depends strongly upon magnetization orientation. Comparing the sensitivity of MOKE for the polar and longitudinal geometry shows a difference by a factor of nearly 6.3. ${ }^{22} \mathrm{We}$ have multiplied the data displayed in Fig. 2 with this value to compare them with the previously reported data for $\mathrm{Fe} / \mathrm{Cu}(100) .{ }^{5}$ For the $\mathrm{Fe} / \mathrm{Cu}(100)$ system a gradient of $235 \pm 15 \mu \mathrm{rad} / \mathrm{ML}$ has been reported in the first thickness range. ${ }^{13}$ If we rescale the observed magnetization for $\mathrm{Fe} / \mathrm{Ni} /$ $\mathrm{Cu}(100)$ with the corresponding sensitivity factor we obtain a gradient of $223 \pm 11 \mu \mathrm{rad}$. This is evidence for a similar magnetic moment of the iron atoms on the $\mathrm{Ni} / \mathrm{Cu}(100)$ substrate.

The magnetization in region II is approximately $300 \mu \mathrm{rad}$ for $\mathrm{Fe} / \mathrm{Cu}(100){ }^{5,13}$ This corresponds to $1.3 \mathrm{ML}$ of ferromagnetic $\mathrm{Fe}$ assuming a similar magnetic moment per atom in regions I and II. Applying this concept to $\mathrm{Fe} / \mathrm{Ni} / \mathrm{Cu}(100)$ as well we derive a magnetic thickness of $0.9 \mathrm{ML}$, again assuming a similar magnetic moment in regions I and II for $\mathrm{Fe} / \mathrm{Ni} /$ $\mathrm{Cu}(100)$. The constant magnetization in region II is not in line with a homogeneously magnetized Fe film. On the contrary, the data can only be explained by a small and constant number of ferromagnetic Fe layers. It is reasonable to assume that these ferromagnetic layers are either located at the film surface or the $\mathrm{Fe} / \mathrm{Ni}$ interface. Without additional measurements, however, it is not possible to identify the position of the ferromagnetic layers. The required additional information can be derived from the temperature dependent measurement displayed in Fig. 3 in conjunction with previous studies. We will start by discussing the magnetization around $300 \mathrm{~K}$. For these temperatures we observe an almost temperature independent magnetization. Previous measurements on both $\mathrm{Ni} / \mathrm{Cu}(100)$ and $\mathrm{Co} / \mathrm{Cu}(100)$ only find a ferromagnetic coupling at the $\mathrm{Fe} / \mathrm{Ni}$ and $\mathrm{Fe} / \mathrm{Co}$ interface at room temperature. Hence, it is very reasonable to assume that the magnetization around $300 \mathrm{~K}$ is only caused by the ferromagnetic Fe layers coupling to the underlying Ni substrate. Since the Ni films have a $T_{c}$ considerably above $300 \mathrm{~K}$, the observed Fe interface magnetization is almost constant around $300 \mathrm{~K}$. The much higher signal at $240 \mathrm{~K}$ can only be explained by an additional contribution. The most plausible assumption is that this contribution comes from ferromagnetic surface layers. $\mathrm{On} \mathrm{Cu}(100)$ the magnetic live surface layers have a $\mathrm{Cu}-$ rie temperature of $270 \mathrm{~K} .^{5,25}$ This would explain the strong decrease of magnetization around $250 \mathrm{~K}$ for $\mathrm{Fe} / \mathrm{Ni} / \mathrm{Cu}(100)$. Indeed, a very similar result has been derived from MXCD (magnetic circular dichroism in $\mathrm{x}$-ray absorption spectroscopy) measurements of $\mathrm{Fe} / \mathrm{Co} / \mathrm{Cu}(100)$ at $110 \mathrm{~K}$. These data have been explained by ferromagnetically live surface and interface layers which couple ferromagnetically. This does not yet explain the almost constant and rather small magnetization up to $200 \mathrm{~K}$ and the step decline above $200 \mathrm{~K}$. The only plausible assumption is a temperature dependent coupling between the two ferromagnetically live layers. At low temperatures the ferromagnetic surface layer couples antiferromagnetically to the ferromagnetic interface layer. This explains why the saturation magnetization is smaller than for $\mathrm{Fe} / \mathrm{Cu}(100)$. Around $200 \mathrm{~K}$ this coupling becomes fairly weak. Interestingly enough, in previous studies of $\mathrm{Fe} /$ $\mathrm{Cu}(100) \mathrm{Li}$ et al. found evidence for an antiferromagnetic coupling in the interior of $\mathrm{Fe}$ films on $\mathrm{Cu}(100)$ in the thickness range between 5 and $10 \mathrm{ML}^{8}$ The Fe films showed a Neél temperature of $200 \mathrm{~K}^{8}{ }^{8}$ At this temperature we observe a strong decrease in magnetization which might be correlated to the disappearing antiferromagnetic coupling.

The resulting model for the magnetic states at different temperatures is displayed in Fig. 3. The model possesses two characteristic features: ferromagnetic $\mathrm{Fe}$ layers at the film surface and the film interface and a temperature dependent coupling. The magnetic order at the interface comes as no surprise. Similar observations have been reported previously for $\mathrm{Fe} / \mathrm{Co} / \mathrm{Cu}(100)($ Refs. 15,17$)$ and $\mathrm{Fe} / \mathrm{Ni} / \mathrm{Cu}(100) .{ }^{14} \mathrm{~A}$ ferromagnetic layer at the surface has previously been found for $\mathrm{Fe} / \mathrm{Co} / \mathrm{Cu}(100) .{ }^{18}$ The same magnetic phase is also found on $\mathrm{Cu}(100)$. There it is accompanied by a $(2 \times 1)$ surface reconstruction and an enlarged atomic volume at the surface. Since the same structure is also found for $\mathrm{Fe} / \mathrm{Ni}(100)$, a magnetic surface layer is also expected in this case. The most intriguing finding is the temperature dependent coupling of the two ferromagnetic layers. In a previous study of $\mathrm{Fe} / \mathrm{Co} /$ $\mathrm{Cu}(100)$ evidence for a ferromagnetic coupling only has been found. ${ }^{18}$ Here we find an antiferromagnetic coupling below $200 \mathrm{~K}$ and a ferromagnetic coupling above $240 \mathrm{~K}$. In addition, the magnetization is strongly reduced between 200 $\mathrm{K}$ and $240 \mathrm{~K}$. This implies that the magnetic coupling between the two ferromagnetic iron layers is temperature dependent. Two different coupling terms, the bilinear coupling and the biquadratic coupling, govern the strength and the sign of the magnetic coupling. Usually the bilinear coupling is the dominating term. A positive bilinear exchange favors an antiparallel (antiferromagnetic) coupling, while a negative bilinear exchange favors ferromagnetic coupling. This suggests that up to $200 \mathrm{~K}$, the magnetic coupling between the iron films is dominated by a positive bilinear exchange while the behavior above $240 \mathrm{~K}$ can be explained by a negative bilinear exchange. Figure 3 (bottom) shows a schematic of the magnetic coupling in the different temperature regimes. Recently a number of studies have addressed the strength and temperature dependence of the bilinear and biquadratic coupling. ${ }^{26,27}$ These studies show for $\mathrm{NiFe} / \mathrm{Cu}$ multilayers a crossover from antiferromagnetic to ferromagnetic coupling as the temperature is lowered below $200 \mathrm{~K} .{ }^{26} \mathrm{In}$ addition, they observe a low-field magnetoresistance minimum which is attributed to an asymmetric canting of the moments away from the applied field. The canting is related to a strong 
biquadratic coupling. Indeed, a pronounced biquadratic coupling between 200 and $240 \mathrm{~K}$ could explain the low level of magnetization observed in this temperature range. A recent study reveals that above the Neél temperature a regime exists where the biquadratic coupling is considerably enhanced. ${ }^{27}$ As mentioned above, a Neél temperature of $200 \mathrm{~K}$ has been observed in previous studies of 5-10 ML Fe films on $\mathrm{Cu}(100)$. This could be correlated with a strong contribution from the biquadratic coupling and would explain the decrease of magnetization above $200 \mathrm{~K}$.

\section{SUMMARY}

Magnetic properties of $\mathrm{Fe}$ films on $\mathrm{Ni} / \mathrm{Cu}(100)$ have been measured between $110 \mathrm{~K}$ and $300 \mathrm{~K}$. With increasing thickness three different magnetic phases are observed which are closely related to the film structure. Above $10 \mathrm{ML}$ a bcc iron film is formed which is homogeneously magnetized and has an in-plane anisotropy. Up to $2.5 \mathrm{ML}$, the Fe film shows a $(4 \times 1)$ superstructure only and is homogeneously magne- tized with in-plane anisotropy as well. The most complex magnetic behavior is found for iron films between 5 and 10 ML. In this thickness region the Fe films are ferromagnetically ordered at the surface. The ferromagnetic surface layers show an enlarged atomic volume and a $(2 \times 1)$ reconstruction, in line with previous findings for $\mathrm{Fe} / \mathrm{Cu}(100)$. Ferromagnetism is also found at the interface while the interior of the Fe film does not show ferromagnetism. Nevertheless, the two ferromagnetic Fe layers couple through the interior of the Fe film. This magnetic coupling shows a strong temperature dependence which leads to both antiferromagnetic and ferromagnetic coupling as well as a canted spin arrangement. This temperature dependence is attributed to the temperature dependence of the bilinear and biquadratic contribution to the exchange coupling.

\section{ACKNOWLEDGMENT}

We gratefully acknowledge financial support by the Deutsche Forschungsgemeinschaft (Wu 243/2).
${ }^{1}$ G. Binasch, P. Grünberg, F. Saurenbach, and W. Zinn, Phys. Rev. B 39, 4828 (1989); M.N. Baibich, J.M. Broto, A. Fert, F. Nguyen van Dau, F. Petroff, P. Etienne, G. Creuzet, A. Friederich, and J. Chazeles, Phys. Rev. Lett. 61, 2472 (1988).

${ }^{2}$ M. Wuttig and B. Feldmann, Surf. Rev. Lett. 3, 1473 (1996).

${ }^{3}$ S. Müller, P. Bayer, C. Reischl, K. Heinz, B. Feldmann, H. Zillgen, and M. Wuttig, Phys. Rev. Lett. 74, 765 (1995).

${ }^{4}$ A. Berger, B. Feldmann, H. Zillgen, and M. Wuttig, J. Magn. Magn. Mater. 183, 35 (1998).

${ }^{5}$ J. Thomassen, F. May, B. Feldmann, M. Wuttig, and H. Ibach, Phys. Rev. Lett. 69, 3831 (1992)

${ }^{6}$ M. Straub, R. Vollmer, and J. Kirschner, Phys. Rev. Lett. 77, 743 (1996).

${ }^{7}$ B. Gubanka, M. Donath, and F. Passek, Phys. Rev. B 54, 11153 (1996).

${ }^{8}$ D. Li, M. Freitag, J. Pearson, Z.Q. Qiu, and S.D. Bader, Phys. Rev. Lett. 72, 3112 (1994).

${ }^{9}$ K. Kalki, D.D. Chambliss, K.E. Johnson, R.J. Wilson, and S. Chiang, Phys. Rev. B 48, 18344 (1993).

${ }^{10}$ J. Giergiel, J. Shen, J. Woltersdorf, A. Kirilyuk, and J. Kirschner, Phys. Rev. B 52, 8528 (1995).

${ }^{11}$ M. Wuttig, B. Feldmann, J. Thomassen, F. May, H. Zillgen, A. Brodde, H. Hannemann, and H. Neddermayer, Surf. Sci. 291, 115 (1993).

${ }^{12}$ R. Lorenz and J. Hafner, Phys. Rev. B 58, 5197 (1998).

${ }^{13}$ B. Feldmann, Ph.D. thesis, Forschungszentrum Jülich, 1996.
${ }^{14}$ W.L. O'Brien, T. Droubay, and B.P. Tonner, Phys. Rev. B 54, 9297 (1996).

${ }^{15}$ W.L. O'Brien and B.P. Tonner, Phys. Rev. B 52, 15332 (1995).

${ }^{16}$ Ernesto J. Escorcia-Aparicio, R.K. Kawakami, and Z.Q. Qiu, J. Appl. Phys. 79, 4964 (1996); Phys. Rev. B 54, 4155 (1996).

${ }^{17}$ R.K. Kawakami, Ernesto J. Escorcia-Aparicio, and Z.Q. Qiu, J. Vac. Sci. Technol. B 14, 3164 (1996).

${ }^{18}$ D. Schmitz, C. Charton, A. Scholl, C. Carbone, and W. Eberhardt, Phys. Rev. B 59, 4327 (1999).

${ }^{19}$ B. Schirmer and M. Wuttig, Surf. Sci. 399, 70 (1998).

${ }^{20}$ J. Thomassen, B. Feldmann, and M. Wuttig, Surf. Sci. 264, 406 (1992).

${ }^{21}$ J. Thomassen, Ph.D. thesis, Forschungszentrum Jülich, 1993.

${ }^{22}$ J. Zak, E.R. Moog, C. Liu, and S.D. Bader, Phys. Rev. B 43, 6423 (1991).

${ }^{23}$ S.Z. Wu, G.J. Mankey, F. Huang, and R.F. Willis, J. Appl. Phys. 76, 6434 (1994).

${ }^{24}$ G. Bochi, C.A. Ballentine, H.E. Inglefield, C.V. Thompson, R.C. O'Handley, H.J. Hug, B. Stiefel, A. Moser, and H.-J. Güntherodt, Phys. Rev. B 52, 7311 (1995).

${ }^{25}$ M. Zharnikov, A. Dittschar, W. Kuch, C.M. Schneider, and J. Kirschner, Phys. Rev. Lett. 76, 4620 (1996); J. Magn. Magn. Mater. 174, 40 (1997).

${ }^{26}$ K. Pettit, S. Gider, S.S.P. Parkin, and M.B. Salamon, Phys. Rev. B 56, 7819 (1997).

${ }^{27}$ E.E. Fullerton, C.H. Sowers, and S.D. Bader, Phys. Rev. B 56, 5468 (1997). 